\title{
Familial small supernumerary marker chromosomes are predominantly inherited via the maternal line
}

\section{To the Editor:}

We read with interest the recently published paper of Dalprà et al. ${ }^{1}$ in this journal. They report on 241 cases with small supernumerary marker chromosomes (sSMC) studied in 19 different Italian laboratories within the last 10 years. This paper is another important contribution to the still understudied field of sSMC-research. Among other interesting conclusions, the authors also report that $16 \%$ of their sSMC cases were maternally inherited and only $7 \%$ paternally inherited. ${ }^{1}$ In their discussion, the authors ${ }^{1}$ allude to a similar 2:1 ratio of maternally/paternally derived sSMC in a previous paper of Crolla et al. ${ }^{2}$ However, no correlation was described or suggested in either a recent paper of Crolla et al. reporting on 137 sSMC-carriers ${ }^{3}$, nor in any previous paper.

This led us to analyze the recently summarized data on all reported sSMC cases to see if such a correlation really exists. By January, 2006, we collected 1,885 sSMC cases on the sSMC-homepage. ${ }^{4}$ One thousand, one hundred eighty-two of these cases were de novo, 102 inherited, and for the remainder, no information on the parental origin was available (Table 1). The following data are derived from the 1,284 cases in which information on the parental origin was available. As known by newborn studies, ${ }^{4-5}$ the incidence of familial sSMC cases is 
Table 1

Parental and chromosomal origin of all reported sSMC cases

\begin{tabular}{|c|c|c|c|c|c|}
\hline Chromosomal origin & Maternal & Paternal & de novo & $\begin{array}{l}\text { N.A., unclear or } \\
\text { neocentromere }\end{array}$ & In summary \\
\hline & \multicolumn{5}{|c|}{ Non-acrocentric chromosomes } \\
\hline 1 & 1 & 0 & 37 & 11 & 49 \\
\hline $1 / 5 / 19$ & 0 & 0 & 5 & 1 & 6 \\
\hline 2 & 1 & 0 & 12 & 8 & 21 \\
\hline 3 & 3 & 0 & 13 & 10 & 26 \\
\hline 4 & 0 & 0 & 15 & 4 & 19 \\
\hline 5 & 0 & 0 & 8 & 6 & 14 \\
\hline 6 & 1 & 0 & 11 & 8 & 20 \\
\hline 7 & 1 & 1 & 14 & 5 & 21 \\
\hline 8 & 1 & 0 & 35 & 18 & 54 \\
\hline 9 & 5 & 0 & 13 & 6 & 24 \\
\hline 10 & 0 & 0 & 8 & 5 & 13 \\
\hline 11 & 0 & 1 & 7 & 2 & 10 \\
\hline 12 & 1 & 0 & 177 & 6 & 184 \\
\hline 16 & 2 & 1 & 17 & 6 & 26 \\
\hline 17 & 0 & 0 & 18 & 4 & 22 \\
\hline 18 & 1 & 0 & 116 & 9 & 126 \\
\hline 19 & 1 & 0 & 20 & 2 & 23 \\
\hline 20 & 1 & 0 & 18 & 7 & 26 \\
\hline $\mathrm{X}$ & 0 & 0 & 16 & 10 & 26 \\
\hline Y & 0 & 2 & 6 & 1 & 9 \\
\hline \multirow[t]{2}{*}{ Summary } & 19 & 5 & 566 & 129 & 719 \\
\hline & \multicolumn{5}{|c|}{ Acrocentric chromosomes } \\
\hline 13 & 1 & 0 & 3 & 10 & 14 \\
\hline $13 / 21$ & 5 & 3 & 26 & 26 & 60 \\
\hline 14 & 8 & 3 & 20 & 35 & 66 \\
\hline $14 / 22$ & 2 & 1 & 12 & 14 & 29 \\
\hline 15 & 20 & 11 & 202 & 329 & 562 \\
\hline 21 & 4 & 0 & 9 & 3 & 16 \\
\hline 22 & 13 & 7 & 344 & 50 & 414 \\
\hline Any acrocentric chromosome & 0 & 0 & 0 & 5 & 5 \\
\hline Summary & 53 & 25 & 616 & 472 & 1166 \\
\hline Overall summary & 72 & 30 & 1182 & 601 & 1885 \\
\hline
\end{tabular}

$40 \%$. At present, only $8 \%$ of the cases were familial. Thus, this data shows that there is a bias in the available data. ${ }^{4}$ Interesting sSMC-cases are reported throughout the literature and sSMC which are not correlated with clinical problems are not that likely to be reported, i.e., sSMC in connection with clinical abnormalities.

However, when posing the question for the relation of maternal-to-paternal inheritance of familial sSMC there is no reason to suggest a similar bias for those 102 cases in which data are available for the parental origin of the sSMC. For those cases (Table 1), we find $72 \mathrm{sSMC}$ inherited from the mother versus 30 sSMC inherited from the father, which is similar to the 2.4:1 ratio suggested in the paper by Dalprà et al. ${ }^{1}$ for the parental origin of sSMC. As detailed in Table 1, this ratio is more expressed in sSMC derived from non-acrocentric chromosomes. The data presented here is independent of that of Dalprà et al., ${ }^{1}$ as there were no details available on their individual patients.

Thus, the observation that familial small supernumerary marker chromosomes (sSMC) are predominantly inherited via the maternal line is substantiated by this analysis. However, an explanation still lacks. One has to suggest that a yet unknown 
Table 2

Gender of the offspring of sSMC-cases as summarized in Table 1

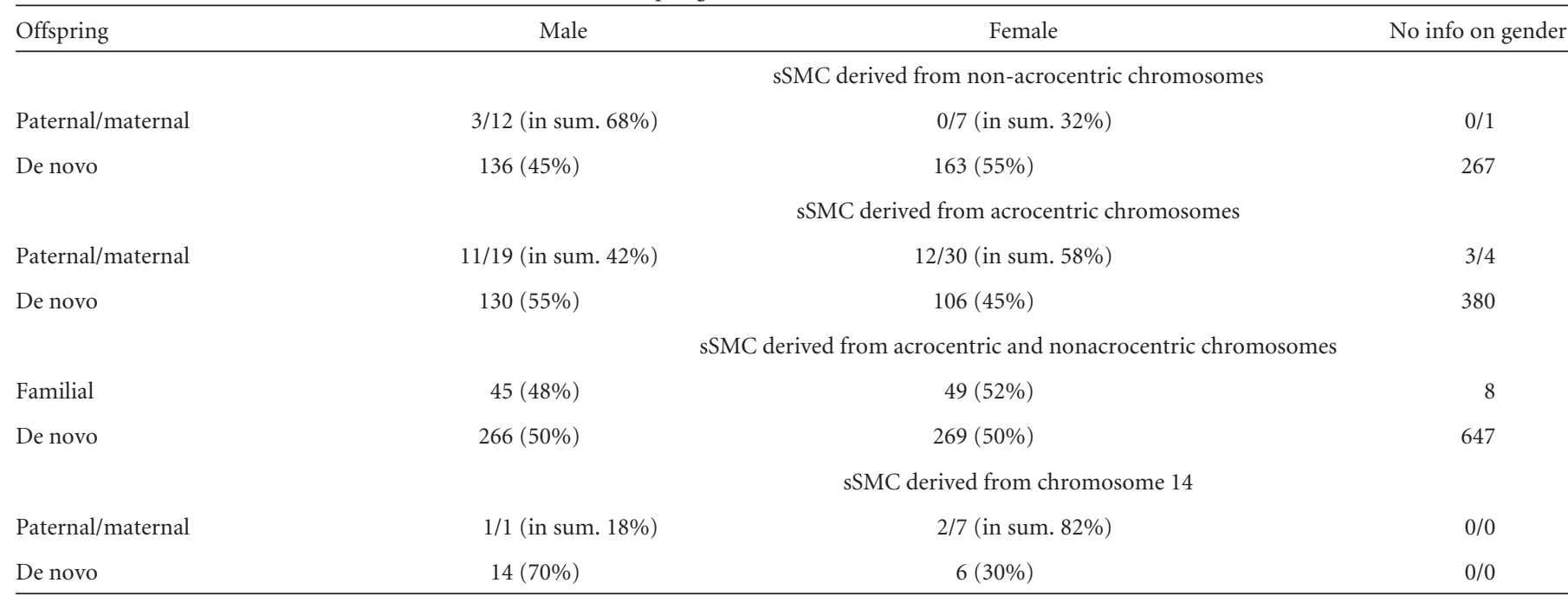

Table 3

Clinical outcome of sSMC-cases as summarized in Table 1

\begin{tabular}{|c|c|c|c|}
\hline Origin of sSMC & No clinical signs & Clinical signs & $\begin{array}{l}\text { No clinical information or } \\
\text { known syndrome like } \\
\text { CES, PKS, i(18p)-, } \\
\text { der(22)- syndrome }\end{array}$ \\
\hline & \multicolumn{3}{|c|}{ sSMC derived from non-acrocentric chromosomes } \\
\hline Paternal/maternal & $2 / 12$ (in sum. $82 \%$ ) & $3 / 4$ (in sum. $18 \%$ ) & $-/ 3$ \\
\hline \multirow[t]{2}{*}{ De novo } & $68(25 \%)$ & $205(75 \%)$ & 293 \\
\hline & \multicolumn{3}{|c|}{ sSMC derived from non-acrocentric chromosomes } \\
\hline Paternal/maternal & $23 / 39$ (in sum. $80 \%$ ) & $1 / 14$ (in sum. $20 \%$ ) & $1 /-$ \\
\hline \multirow[t]{2}{*}{ De novo } & $59(25 \%)$ & $178(75 \%)$ & 379 \\
\hline & \multicolumn{3}{|c|}{ sSMC derived from acrocentric and non-acrocentric chromosomes } \\
\hline Familial & $76(76 \%)$ & $22(22 \%)$ & 4 \\
\hline De novo & $127(25 \%)$ & $383(75 \%)$ & 672 \\
\hline
\end{tabular}

effect is driving selection via fertilization-success of sperm without an sSMC. Problems in connection with sSMC-replication arising predominantly in the more rapidly progressing sperm meiosis or a 'weight-effect' making sperm without more rapid than those with an sSMC, similar to the effect known from Y-versus X-chromosome carrying sperm ${ }^{6}$ could be envisaged as possible mechanisms involved here.

The next question that we had based on the available data ${ }^{4}$ was if there is a difference in sex ratio in the offspring coming from the parentally inherited compared to de novo sSMC cases. As summarized in Table 2, 94 familial and 535 de novo cases were available for this problem. There was not enough data to distinguish between maternally and paternally inherited ones for that question. When looking at the overall summary of Table 2 there is a 1:1 sex ratio in the offspring of de novo (266:269) and familial cases (45:49). However, splitting into acrocentric and non-acrocentric derived sSMC there is a difference in the latter familial cases (male-to-female ratio of $2: 1$ ). Though, this could easily be a bias due to low case numbers ( 22 cases). Moreover, it could be that single chromosomes form exceptions here, as shown for chromosome 14 in Table 2: among 11 parentally inherited sSMC (14) cases, only $20 \%$ were male offspring, while in the 20 available de novo sSMC (14) cases $70 \%$ were male. Further studies are required to clarify this point.

As summarized in Table 3, there is a difference in the clinical outcome of familial versus de novo cases. While $76 \%$ of the familial cases are without clinical signs, $75 \%$ of the reported de novo cases show clinical abnormalities. However, for this question one, has to admit that the bias is valid, that predominantly 'interesting' cases are reported in the literature. As summarized on the sSMC-homepage ${ }^{4}$ it is known by studies performed in newborn cohorts, ${ }^{7-9}$ that de novo sSMC are associated with clinical signs only in $11-30 \%$. Thus, the only 
conclusions to be drawn from this data are that a small but considerable part of familial sSMC can lead to clinical problems in the offspring. This has to be considered in genetic counseling of familial cases as well. Moreover, there was a first genotype-phenotype correlation for sSMC published recently, ${ }^{10}$ which can be considered in clinical cases, too.

In summary, the analysis of all in detail reported sSMC cases $^{4}$ concerning the differentiation of maternal and paternal origin, sex ratio, and clinical signs of the offspring gave the following results. As suggested by Dalprà et al. ${ }^{1}$ in this journal, there is a yet unexplained doubled transmittance rate of sSMC via the maternal compared to the paternal line. Apart from chromosome 14 it seems quite clear, that there is a 1:1 sex ratio in the offspring of familial and de novo sSMC cases. Finally, clinical signs in connection with an sSMC are to be expected in a subset of parentally inherited as well as de novo cases.

Thomas Liehr, PhD

Institute of Human Genetics and Anthropology, Kollegiengasse 10, 07743 Jena, Germany

\section{ACKNOWLEDGMENTS}

Supported in parts by the Dr. Robert Pfleger-Stiftung, the DFG (436 RUS 17/135/03; 436 RUS 17/109/04, 436 WER 17/ $1 / 04,436$ WER $17 / 5 / 05,436$ RUS 17/22/06), the DAAD, the EU
(Marie-Curie-Fellowship HPMT-CT-2001-00273), the Schering Foundation and the Evangelische Studienwerk e.V. Villigst.

\section{References}

1. Dalpà L, Giardino D, Finelli $\mathrm{P}$, Corti C, et al. Cytogenetic and molecular evaluation of 241 small supernumerary marker chromosomes: cooperative study of 19 Italian laboratories. Genet Med 2005;7:620-625.

2. Crolla JA, Long F, Rivera H, Dennis NR. FISH and molecular study of autosomal supernumerary marker chromosomes excluding those derived from chromosomes 15 and 22: I. Results of 26 new cases. Am J Med Genet 1998;75:355-366.

3. Crolla JA, Youings SA, Ennis S, Jacobs PA. Supernumerary marker chromosomes in man: parental origin, mosaicism and maternal age revisited. Eur J Hum Genet 2005; 13:154-160.

4. Liehr T. sSMC homepage Available at: http://mti-n.mti.uni-jena.de/ huwww/ MOL_ZYTO/sSMC/00START.htm, Accessed on: January 19, 2006.

5. Liehr T, Claussen U, Starke H. Small supernumerary marker chromosomes (sSMC) in humans. Cytogenet Genome Res 2004;107:55-67.

6. Smits LJ, de Bie RA, Essed GG, van den Brandt PA. Time to pregnancy and sex of offspring: cohort study. BMJ 2005;331:1437-1438.

7. Daniel A, Malafiej P. A Series of supernumerary small ring marker autosomes identified by FISH with chromosome probe arrays and literature review excluding chromosome 15. Am J Med Genet 2003;117:A212-A222.

8. Warburton D. De novo balanced chromosome rearrangements and extra marker chromosomes identified at prenatal diagnosis: clinical significance and distribution of breakpoints. Am J Hum Genet 1991;49:995-1013.

9. Crolla JA. FISH and molecular studies of autosomal supernumerary marker chromosomes excluding those derived from chromosome 15: II. Review of the literature. Am J Med Genet 1998;75:367-381.

10. Liehr T, Mrasek K, Weise A, Dufke A, et al. Small supernumerary marker chromosomes-progress towards a genotype-phenotype correlation. Cytogenet Genome Res 2006;112:23-34. 